\title{
Two-meson cloud contribution to the baryon antidecuplet binding
}

\author{
A. Hosaka, ${ }^{1}$ T. Hyodo, ${ }^{1, *}$ F. J. Llanes-Estrada, ${ }^{2, \dagger}$ E. Oset,${ }^{3}$ J. R. Peláez, ${ }^{4}$ and M. J. Vicente Vacas ${ }^{3}$ \\ ${ }^{1}$ Research Center for Nuclear Physics (RCNP), Ibaraki, Osaka 567-0047, Japan \\ ${ }^{2}$ Universidad Complutense de Madrid, Depto. Física Teórica I, E-28040 Madrid, Spain \\ ${ }^{3}$ Departamento de Física Teórica and IFIC, Centro Mixto Universidad de Valencia-CSIC, \\ Institutos de Investigación de Paterna, Aptd. 22085, E-46071 Valencia, Spain \\ ${ }^{4}$ Universidad Complutense de Madrid, Depto. Física Teórica II, E-28040 Madrid, Spain
}

(Received 1 December 2004; published 21 April 2005)

\begin{abstract}
We study the two-meson virtual cloud contribution to the self-energy of the SU(3) antidecuplet, to which the $\Theta^{+}$pentaquark is assumed to belong. This is motivated by the large branching ratio of the $N(1710)$ decay into two pions and one nucleon. We derive effective Lagrangians that describe the $N(1710)$ decay into $N \pi \pi$ with two pions in $s$ or $p$ wave. We obtain increased binding for all members of the antidecuplet and a contribution to the mass splitting between states with different strangeness which is at least $20 \%$ of the empirical one. We also provide predictions for three-body decays of the pentaquark antidecuplet.
\end{abstract}

DOI: 10.1103/PhysRevC.71.045205

PACS number(s): 14.20.-c, 11.30.Hv, 12.40.Yx, 24.85.+p

\section{INTRODUCTION}

The observation of the signal of the $\Theta^{+}$exotic baryon [1], broadly known as the pentaquark for its minimal fivequark Fock space assignment in quantum chromodynamics, was stimulated by the prediction $[2,3]$ of a $1 / 2^{+}$baryon antidecuplet. Many experimental and theoretical studies have been devoted to this resonance [4,5]. The original states proposed to form this antidecuplet [2] were

$$
\Theta(1540), N(1710), \Sigma(1890), \Xi(2070),
$$

where the first and last, explicitly exotic states, had not been observed at that time. Isospin $\mathrm{SU}(2)$ is expected to hold to very good accuracy, and we do not list the $I_{3}$ quantum number.

This assignment is now, however, being challenged for several reasons. First, the NA49 Collaboration [6] reported evidence for an exotic cascade $\Xi^{--}$, probably in the same antidecuplet, with a much lighter mass, $1860 \mathrm{MeV}$. This is somewhat problematic as doubts have arisen [7], and it has not been seen in other experiments [8,9]. (See, however, K. Kadija's presentation at the PENTAQUARK04 Workshop [10] with new reanalyses still supporting the findings of Ref. [7]). However, should the state be reconfirmed at $1860 \mathrm{MeV}$, using the standard Gell-Mann-Okubo rule (GMO) of equal mass splittings for the SU(3) antidecuplet, the mass of $N_{\overline{10}}$ would have to be near $1647 \mathrm{MeV}$, which is about $60 \mathrm{MeV}$ below the nominal one $N(1710)$. Furthermore, the mass of $\Sigma_{\overline{10}}$ would have to be about $1753 \mathrm{MeV}$. Since a $\Sigma$ resonance is listed at $1770 \mathrm{MeV}$ with the same spin and parity, we will refer to the $\Sigma$ member of the antidecuplet as $\Sigma(1770)$. The association of this state to the $\Sigma(1890)$ would not fit in that scenario [11].

Second, quark model calculations that have appeared after the report of the evidence of $\Theta^{+}$tend to predict an $N_{\overline{10}}$ at around

*Electronic address: hyodo@rcnp.osaka-u.ac.jp

†Electronic address: fllanes@fis.ucm.es
$1650 \mathrm{MeV}[12,13]$. These predicted the $\Xi_{\overline{10}}$ at $1900 \mathrm{MeV}$ [13], which is more in line with the experimental outcome than the original calculation of the chiral soliton model, although the latter can be readjusted (then underestimating the $N^{*}$ ).

Another difficulty arises because of the potential mixing of the nonexotic members of the multiplet, the $N_{\overline{10}}$ and $\Sigma_{\overline{10}}$ with members of pentaquark or ordinary three-quark octets $[11,14,15]$. This would make the mass splitting between the physical states dependent on two mixing angles. A current conjecture is a mixing with the Roper resonance [14] that would, by level repulsion, push the $N(1710)$ farther above the $\Theta^{+}$than predicted by the GMO rule. Also, with ideal mixing, the hidden strangeness $s \bar{s}$ wave function dominates the $N(1710)$, thus raising its mass. However, such a strong mixing is not preferred by other authors [11,16,17]. In summary, a new $N^{*}$ state would have to be searched for at a smaller mass if we were to impose perfect GMO rule.

The models we work with in this paper are rather phenomenological. However, our method, based on symmetry principles, is suited to at least estimating meson cloud effects, which are important for the understanding of pentaquark properties. The main conclusion of this work is that the virtual "two-meson cloud" yields an attractive self-energy that provides about $20 \%$ of the pentaquark mass splittings. We believe that our study here will become useful when more data are available.

The study presented here is complementary and looks for another source of mass splitting not contemplated by the GMO rule. It would come from the two-meson cloud. The possibility of constructing the $\Theta^{+}$as a $K \pi N$ bound state [18-21] has been examined in some detail [22] employing meson-meson and meson-baryon interactions from chiral Lagrangians, where attraction was found but not strong enough to bind the system. Yet, this result leaves one wondering as to what role the twomeson cloud could play in the stability of the state. Coupling to multimeson components is also implicit in the chiral soliton picture, which leads to small masses of the $\Theta^{+}[2,23]$.

In the present paper, we do not face the possible contribution of the one-meson cloud to the antidecuplet binding, which can 
be easily addressed as a minor correction to our results. The small width of the $\Theta^{+}$to $K N$, in spite of the appreciable phase space available, qualitatively demands that this contribution should be reasonably small; in fact, it has been checked quantitatively in Refs. [24-26]. The self-energy of $\Theta^{+}$with a two-meson cloud has been studied in parallel [26] in the context of the medium modification of $\Theta^{+}$and possible formation of $\Theta^{+}$hypernuclei [27]. We here report in full on vacuum results for not only the $\Theta^{+}$but also other members of the antidecuplet.

An important experimental input relevant to the present study is the relatively large branching ratio of $N(1710)$ into $N \pi \pi$, about 40-90\% [28]. The branching ratio into $N \pi \pi$ with the two pions in an $s$ wave is $10-40 \%$ and into $\rho N, 5-25 \%$. This $N(1710)$ resonance and its baryon-meson-meson decay mode has been used in Ref. [29] to produce a good shape of the $\Sigma \pi$ distribution in the $\pi^{-} p \rightarrow K^{0} \pi \Sigma$ reaction leading to the $\Lambda(1405){ }^{1}$

In the present work, we assume that the $N(1710)$ has a large antidecuplet component [11], and we will perform a study of the $N \pi \pi s$ wave and the $\rho N$ decay channels of this resonance and their influence on the masses of various members of the antidecuplet. Certainly one has to accept a mixing with an octet component for realistic resonances in order, for instance, to explain the $N(1710)$ decay into $\Delta \pi$, which is forbidden for its antidecuplet component $[15,30]$. But we do not expect the mixing angle to be close to ideal, as this would imply a stronger $\Lambda K$ branching ratio than $5-25 \%$, as observed experimentally. The decay pattern of $N(1710)$ and $N(1440)$ also supports the small mixing angle $[16,17]$.

The present study also provides information on the pentaquark (antidecuplet baryon)-baryon-meson-meson (PBMM) contact interaction, which could be applied to the study of $\Theta^{+}$production with the $\pi^{-} p \rightarrow K^{-} \Theta^{+}$and $K^{+} p \rightarrow$ $\pi^{+} \Theta^{+}$reactions. These reactions are studied in Refs. [31-34] and experimental information is becoming available [35,36].

The paper is organized as follows. In Sec. II, we construct various $P B M M$ interactions with the two octet mesons and one baryon belonging to octets and with the other baryon to an antidecuplet. In Sec. III, we compute the contributions of two-meson and one-baryon loops to the mass splittings among the members of antidecuplet baryons. In Sec. IV, we present numerical results and discuss the importance of two-meson contributions to the mass splittings and partial decay widths. As we will see, the contributions from the two-meson loops provide sizable contributions to supplement the mass splittings naively expected from strange quark counting. We will then discuss the range of interaction strengths of various coupling terms. Section V is devoted to a summary. We also add appendices, where complete tables for the PBMM interactions are presented.

\footnotetext{
${ }^{1}$ In that work, the $N(1710)$ was assumed to belong to an octet representation, although such a selection is not crucial to the results obtained there.
}

\section{CONSTRUCTION OF EFFECTIVE INTERACTION LAGRANGIANS}

\section{A. Definition of fields}

Following a common convention [37-39], we write the physical meson and baryon fields as follows

$$
\begin{aligned}
\phi & =\left(\begin{array}{ccc}
\frac{1}{\sqrt{2}} \pi^{0}+\frac{1}{\sqrt{6}} \eta & \pi^{+} & K^{+} \\
\pi^{-} & -\frac{1}{\sqrt{2}} \pi^{0}+\frac{1}{\sqrt{6}} \eta & K^{0} \\
K^{-} & \bar{K}^{0} & -\frac{2}{\sqrt{6}} \eta
\end{array}\right), \\
B & =\left(\begin{array}{ccc}
\frac{1}{\sqrt{2}} \Sigma^{0}+\frac{1}{\sqrt{6}} \Lambda & \Sigma^{+} & p \\
\Sigma^{-} & -\frac{1}{\sqrt{2}} \Sigma^{0}+\frac{1}{\sqrt{6}} \Lambda & n \\
\Xi^{-} & \Xi^{0} & -\frac{2}{\sqrt{6}} \Lambda
\end{array}\right) .
\end{aligned}
$$

The antidecuplet containing the exotic pentaquark states is a tensor $P^{i j k}$ totally symmetric in its three SU(3) indices. The components of $P^{i j k}$ are related to the physical fields by

$$
\begin{array}{ll}
P^{333}=\sqrt{6} \Theta_{\frac{+}{10},}^{+} & P^{133}=\sqrt{2} N_{\overline{10}}^{0}, \\
P^{233}=-\sqrt{2} N_{\overline{10}}^{+}, & P^{113}=\sqrt{2} \Sigma_{\overline{10}}^{-}, \\
P^{123}=-\Sigma_{\overline{10}}^{0}, & P^{223}=-\sqrt{2} \Sigma_{\overline{10}}^{+}, \\
P^{111}=\sqrt{6} \Xi_{\overline{10}}^{--}, & P^{112}=-\sqrt{2} \Xi_{\overline{10}}^{-}, \\
P^{122}=\sqrt{2} \Xi_{\overline{10}}^{0}, & P^{222}=-\sqrt{6} \Xi_{\overline{10}}^{+},
\end{array}
$$

where we have adopted the normalization in Ref. [40], which is different from those used in Refs. $[25,41,42]$ by a sign and/or a factor.

Now we consider the possible interaction Lagrangians, constrained to be $\mathrm{SU}(3)$ symmetric. We intend to address the process

$$
\mathbf{8}_{M}+\mathbf{8}_{M}+\mathbf{8}_{B} \rightarrow \overline{\mathbf{1 0}}_{P}
$$

where an octet baryon $\mathbf{8}_{B}$ and two octet mesons $\mathbf{8}_{M}$ couple to an antidecuplet baryon $\overline{\mathbf{1 0}}_{P}$. To have an $\mathrm{SU}(3)$ invariant Lagrangian, we couple first the two $\mathbf{8}_{M}$ and then combine the resulting irreducible representations with the baryon $\mathbf{8}_{B}$ to produce a $\overline{\mathbf{1 0}}_{B M M}$ representation. The group theoretical irreducible decomposition gives

$$
\begin{aligned}
\mathbf{8}_{M} & \otimes \mathbf{8}_{M} \otimes \mathbf{8}_{B} \\
= & \left(\mathbf{1} \oplus \mathbf{8}^{s} \oplus \mathbf{8}^{a} \oplus \mathbf{1 0} \oplus \overline{\mathbf{1 0}} \oplus \mathbf{2 7}\right)_{M M} \otimes \mathbf{8}_{B} \\
= & \mathbf{8} \leftarrow \text { from } \mathbf{1}_{M M} \otimes \mathbf{8}_{B} \\
& \oplus(\mathbf{1} \oplus \mathbf{8} \oplus \mathbf{8} \oplus \mathbf{1 0} \oplus \overline{\mathbf{1 0}} \oplus \mathbf{2 7}) \leftarrow \text { from } \mathbf{8}_{M M}^{s} \otimes \mathbf{8}_{B} \\
& \oplus(\mathbf{1} \oplus \mathbf{8} \oplus \mathbf{8} \oplus \mathbf{1 0} \oplus \overline{\mathbf{1 0}} \oplus \mathbf{2 7}) \leftarrow \text { from } \mathbf{8}_{M M}^{a} \otimes \mathbf{8}_{B} \\
& \oplus(\mathbf{8} \oplus \mathbf{1 0} \oplus \mathbf{2 7} \oplus \mathbf{3 5}) \leftarrow \text { from } \mathbf{1 0}_{M M} \otimes \mathbf{8}_{B} \\
& \oplus(\mathbf{8} \oplus \overline{\mathbf{1 0}} \oplus \mathbf{2 7} \oplus \mathbf{3 5}) \leftarrow \text { from } \overline{\mathbf{1 0}}_{M M} \otimes \mathbf{8}_{B} \\
& \oplus\left(\mathbf{8} \oplus \mathbf{1 0} \oplus \overline{\mathbf{1 0}} \oplus \mathbf{2 7} \oplus \mathbf{2 7} \oplus \mathbf{3 5} \oplus \mathbf{3 5}^{\prime \prime} \oplus \mathbf{6 4}\right) \\
& \leftarrow \text { from } \mathbf{2 7} 7_{M M} \otimes \mathbf{8}_{B} .
\end{aligned}
$$

Here $\mathbf{8}^{s}$ and $\mathbf{8}^{a}$ denote symmetric and antisymmetric combinations of the two-meson fields. Hence, we obtain four $\overline{\mathbf{1 0}}_{B M M}$ representations after recoupling $\mathbf{8}_{M M}^{s}, \mathbf{8}_{M M}^{a}, \mathbf{1 0}_{M M}$, and $27_{M M}$ with $\mathbf{8}_{B}$. 


\section{B. Two-meson $8^{s}$ representation}

In constructing effective Lagrangians, we follow the principle of using the minimum number of derivatives in the fields. This will be released later when we discuss possible structures involving derivatives. To construct $\mathbf{8}^{s}$ from two $\mathbf{8}_{M}$, we have in tensor notation

$$
\begin{aligned}
D_{i}{ }^{j}\left[\mathbf{8}_{M M}^{s}\right] & =\phi_{i}{ }^{a} \phi_{a}{ }^{j}+\phi_{i}{ }^{a} \phi_{a}{ }^{j}-\frac{2}{3} \delta_{i}{ }^{j} \phi_{a}{ }^{b} \phi_{b}{ }^{a} \\
& =2 \phi_{i}{ }^{a} \phi_{a}{ }^{j}-\frac{2}{3} \delta_{i}{ }^{j} \phi_{a}{ }^{b} \phi_{b}{ }^{a} .
\end{aligned}
$$

We combine this now with an $\mathbf{8}_{B}$ to give an antidecuplet

$$
\begin{aligned}
T^{i j k}\left[\overline{\mathbf{1 0}}_{B M M(8 s)}\right]= & 2 \phi_{l}{ }^{a} \phi_{a}{ }^{i} B_{m}{ }^{j} \epsilon^{l m k} \\
& +(i, j, k \text { symmetrized }) .
\end{aligned}
$$

Hence, the interaction Lagrangian becomes

$$
\mathcal{L}^{8 s}=\frac{g^{8 s}}{2 f} \bar{P}_{i j k} \epsilon^{l m k} \phi_{l}^{a} \phi_{a}{ }^{i} B_{m}{ }^{j}+\text { h.c. },
$$

where h.c. denotes the Hermitian conjugate terms, in order to take into account the processes in which the antidecuplet is in the initial state. Note also that two $\phi$ fields have appeared, and we have included a factor $1 / 2 f$ in order to make $g^{8 s}$ dimensionless ( $f$ is the pion decay constant $f=93 \mathrm{MeV}$ ).

\section{Two-meson $8^{a}$ representation}

Next we take the antisymmetric combination of the $\mathbf{8}_{M}$ and $\mathbf{8}_{M}$, which for identical meson octets leads to

$$
A_{i}^{j}\left[\mathbf{8}_{M M}^{a}\right]=\phi_{i}{ }^{a} \phi_{a}^{j}-\phi_{i}^{a} \phi_{a}^{j}=0 .
$$

So given the identity of the meson octets, this combination is zero. The simplest way to construct the Lagrangian of this structure is to introduce a derivative in one of the fields, which leads automatically to the vector current consisting of two meson fields. Proceeding as before, we combine this structure with $\mathbf{8}_{B}$ to give $\overline{\mathbf{1 0}}$, then finally

$$
\mathcal{L}^{8 a}=i \frac{g^{8 a}}{4 f^{2}} \bar{P}_{i j k} \epsilon^{l m k} \gamma^{\mu}\left(\partial_{\mu} \phi_{l}{ }^{a} \phi_{a}{ }^{i}-\phi_{l}{ }^{a} \partial_{\mu} \phi_{a}{ }^{i}\right) B_{m}{ }^{j}+\text { h.c. },
$$

where $g^{8 a}$ is dimensionless. This interaction Lagrangian contains the coupling of the $N(1710)$ with $N \pi \pi$, the two pions in a $\rho$-meson type correlation. From the experimental branching ratio, we can determine the coupling constant $g^{8 a}$.

\section{Two-meson $\overline{\mathbf{1 0}}$ representation}

To construct the $\overline{\mathbf{1 0}}$ combination from two mesons, we have now

$$
\begin{aligned}
T_{M M}^{i j k}\left[\overline{\mathbf{1 0}}_{M M}\right]= & \epsilon^{l m k} \phi_{l}{ }^{i} \phi_{m}{ }^{j}+(i, j, k \text { symmetrized }) \\
= & \epsilon^{l m k} \phi_{l}{ }^{i} \phi_{m}{ }^{j}+\epsilon^{l m k} \phi_{l}{ }^{j} \phi_{m}{ }^{i}+\epsilon^{l m i} \phi_{l}{ }^{j} \phi_{m}{ }^{k} \\
& +\epsilon^{l m i} \phi_{l}{ }^{k} \phi_{m}{ }^{j}+\epsilon^{l m j} \phi_{l}{ }^{k} \phi_{m}{ }^{i}+\epsilon^{l m j} \phi_{l}{ }^{i} \phi_{m}{ }^{k} \\
= & 0,
\end{aligned}
$$

which is identically zero for equal meson octets.

\section{E. Two-meson 27 representation}

The expansion for the $\mathbf{2 7}$ representation leads to

$$
\begin{aligned}
H_{i k}^{j l}\left[27_{M M}\right]= & \phi_{i}{ }^{j} \phi_{k}{ }^{l}+\phi_{i}{ }^{l} \phi_{k}{ }^{j}+\phi_{k}{ }^{j} \phi_{i}{ }^{l}+\phi_{k}{ }^{l} \phi_{i}{ }^{j} \\
& -\frac{1}{5}\left(\delta_{i}^{j} D_{k}{ }^{l}+\delta_{i}{ }^{l} D_{k}{ }^{j}+\delta_{k}{ }^{j} D_{i}{ }^{l}+\delta_{k}{ }^{l} D_{i}{ }^{j}\right) \\
& -\frac{1}{6}\left(\delta_{i}{ }^{j} \delta_{k}{ }^{l} \phi_{a}{ }^{b} \phi_{b}{ }^{a}+\delta_{i}{ }^{l} \delta_{k}{ }^{j} \phi_{a}{ }^{b} \phi_{b}{ }^{a}\right),
\end{aligned}
$$

where $D_{i}{ }^{j}$ is defined in Eq. (6). Now the combination of $27_{M M}$ to $\mathbf{8}_{B}$ to give the $\overline{\mathbf{1 0}}$ representation leads to

$$
\begin{aligned}
\mathcal{L}^{27}= & \frac{g^{27}}{2 f}\left[4 \bar{P}_{i j k} \epsilon^{l b k} \phi_{l}{ }^{i} \phi_{a}{ }^{j} B_{b}{ }^{a}\right. \\
& \left.-\frac{4}{5} \bar{P}_{i j k} \epsilon^{l b k} \phi_{l}{ }^{a} \phi_{a}{ }^{j} B_{b}{ }^{i}\right]+ \text { h.c. },
\end{aligned}
$$

where the first term gives us a new $\mathrm{SU}(3)$ structure, but the second one is equal to the $\mathcal{L}^{8 s}$ given in Eq. (8).

To summarize briefly, for the possible SU(3) symmetric couplings of $P B M M$, there are two independent terms with no derivatives, namely Eqs. (8) and (13). With one derivative, there are four more terms available, but we will consider only Eq. (10), which has the structure for the decay of $N(1710) \rightarrow$ $N \pi \pi$ ( $p$ wave) as observed experimentally.

\section{F. Chiral symmetric Lagrangians}

In the perturbative chiral Lagrangian approach, one would like to implement chiral symmetry as a derivative expansion. In addition, one of the advantages of chiral Lagrangians is that they relate coupling constants of different processes and, in particular, with increasing number of mesons. However, in the present case we cannot take advantage of any of these relations, since the couplings for the present Lagrangians are a priori completely arbitrary, and we are only interested in the twomeson problem. Still, in this section we build the lowest-order chiral Lagrangian, with two derivatives. Let us remark that the chiral expansion with baryons is known to converge much more slowly than chiral perturbation theory with mesons, and this lowest-order Lagrangian can only be expected to give a mere qualitative description of the physics. For that reason, to build the Lagrangians of the previous Secs. II B and II E we just relied on flavor SU(3). Still, we will check here that the lack of chiral symmetry in those Lagrangians does not have much relevance to the mass splittings and decays we are interested in, since already with the leading-order Lagrangian we get qualitatively the same results. In other words, the relevant symmetry here is $\mathrm{SU}(3)$, not chiral symmetry.

To show this, we write a chiral invariant Lagrangian by making the substitution $\phi \cdot \phi \rightarrow A_{\mu} \cdot A^{\mu}$ in Eq. (8) such that

$$
\mathcal{L}^{\chi}=\frac{g^{\chi}}{2 f} \bar{P}_{i j k} \epsilon^{l m k}\left(A_{\mu}\right)_{l}{ }^{a}\left(A^{\mu}\right)_{a}{ }^{i} B_{m}{ }^{j}+\text { h.c. },
$$

where $A_{\mu}$ is the axial current written in terms of the chiral field $\xi$,

$$
A_{\mu}=\frac{i}{2}\left(\xi^{\dagger} \partial_{\mu} \xi-\xi \partial_{\mu} \xi^{\dagger}\right)
$$


with $\xi=e^{i \phi / \sqrt{2} f}$. To the leading order in the meson field, $A_{\mu} \sim-\partial_{\mu} \phi / \sqrt{2} f$, we find the interaction Lagrangian induced from Eq. (14) by making the replacement

$$
\left(A_{\mu}\right)_{l}{ }^{a}\left(A^{\mu}\right)_{a}{ }^{i} \rightarrow \frac{1}{2 f^{2}} \partial_{\mu} \phi_{l}{ }^{a} \partial^{\mu} \phi_{a}{ }^{i} .
$$

Obviously, the SU(3) structure is not affected by this procedure, although the use of Lagrangians involving derivatives will introduce some degree of SU(3) breaking due to the momenta of mesons. Hence, it is useful to verify that this chiral invariant Lagrangian will lead eventually to the same results as those obtained from the Lagrangians without derivatives in the fields. We also perform self-energy calculations using this $\mathbf{8}^{s}$ chirally symmetric Lagrangian, Eq. (14).

\section{G. Explicit SU(3) breaking term}

In this section, we consider the $\mathrm{SU}(3)$ breaking interaction term within the context of chiral Lagrangians. Without using derivatives in the fields, the only possible term is a mass term that violates both SU(3) and chiral symmetry, but in the way demanded by the underlying QCD Lagrangian [37-39]. The mass term appears through the combination

$$
S=\xi M \xi+\xi^{\dagger} M \xi^{\dagger},
$$

with the mass matrix, written in terms of the meson masses,

$$
M=\left(\begin{array}{ccc}
m_{\pi}^{2} & & \\
& m_{\pi}^{2} & \\
& & 2 m_{K}^{2}-m_{\pi}^{2}
\end{array}\right) .
$$

Then it leads to the Lagrangian

$$
\mathcal{L}^{M}=\frac{g^{M}}{2 f} \bar{P}_{i j k} \epsilon^{l m k} S_{l}^{i} B_{m}^{j}+\text { h.c. }
$$

In the expansion of $S$, we have two meson fields with the structure

$$
S^{(2)}=-\frac{1}{2 f^{2}}(2 \phi M \phi+\phi \phi M+M \phi \phi) .
$$

Substituting $S^{(2)}$ for $S$ in Eq. (19), we obtain the desired mass Lagrangian.

\section{SELF-ENERGIES}

\section{A. Two-meson loops}

The antidecuplet self-energies deduced from one of the interaction Lagrangians can be obtained by

$$
\begin{aligned}
\Sigma_{P}^{(j)}\left(p^{0}\right)= & \sum_{B, m_{1}, m_{2}}\left(F^{(j)} C_{P, B, m_{1}, m_{2}}^{(j)}\right) \\
& \times I^{(j)}\left(p^{0} ; B, m_{1}, m_{2}\right)\left(F^{(j)} C_{P, B, m_{1}, m_{2}}^{(j)}\right),
\end{aligned}
$$

where the index $j$ stands for $8 s, 8 a, 27, \chi$, and $M$ for corresponding Lagrangians (8), (10), (13), (14), and (19); $P$ denotes the antidecuplet states $P=\Theta_{\overline{10}}, N_{\overline{10}}, \Sigma_{\overline{10}}$, and $\Xi_{\overline{10}}$; the argument $p^{0}$ is the energy of the antidecuplet baryon; and

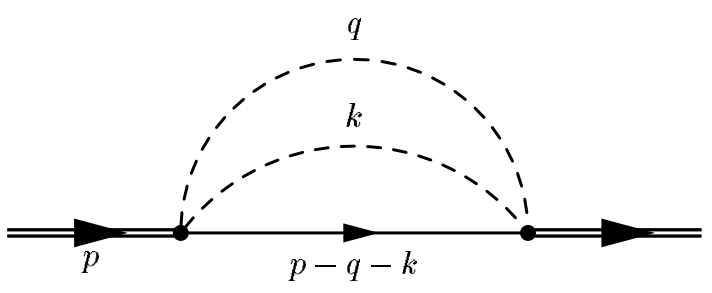

FIG. 1. Self-energy of baryon antidecuplet caused by two-meson cloud.

the factors $F^{(j)}$ are

$$
\begin{gathered}
F^{8 s}=\frac{g^{8 s}}{2 f}, \quad F^{8 a}=\frac{g^{8 a}}{4 f^{2}}, \\
F^{27}=\frac{g^{27}}{2 f}, \quad F^{\chi}=\frac{g^{\chi}}{2 f}, \quad F^{M}=\frac{g^{M}}{2 f} .
\end{gathered}
$$

In Eq. (21), $C_{P, B, m_{1}, m_{2}}^{(j)}$ are $\mathrm{SU}(3)$ coefficients that come directly from the Lagrangians when evaluating the different matrix elements. We compile the results in App. A.

The function $I^{(j)}\left(p^{0} ; B, m_{1}, m_{2}\right)$ of argument $p^{0}$ (the energy of the assumed state of the antidecuplet at rest) is the two-loop integral with two mesons and one baryon as shown in Fig. 1.

$$
\begin{aligned}
I^{(j)}\left(p^{0} ; B, m_{1}, m_{2}\right)= & -\int \frac{d^{4} k}{(2 \pi)^{4}} \int \frac{d^{4} q}{(2 \pi)^{4}} \\
& \times\left|t^{(j)}\right|^{2} \frac{1}{k^{2}-m_{1}^{2}+i \epsilon} \frac{1}{q^{2}-m_{2}^{2}+i \epsilon} \\
& \times \frac{M}{E} \frac{1}{p^{0}-k^{0}-q^{0}-E+i \epsilon},
\end{aligned}
$$

where

$$
\begin{aligned}
\left|t^{(j)}\right|^{2}= & 1 \quad \text { for } \quad j=8 s, 27, M \\
\left|t^{\chi}\right|^{2}= & \frac{\left(\omega_{1} \omega_{2}-\boldsymbol{k} \cdot \boldsymbol{q}\right)^{2}}{4 f^{4}} \\
\left|t^{8 a}\right|^{2}= & \frac{1}{2 M}\left\{(E+M)\left(\omega_{1}-\omega_{2}\right)^{2}+2\left(|\boldsymbol{k}|^{2}-|\boldsymbol{q}|^{2}\right)\left(\omega_{1}-\omega_{2}\right)\right. \\
& \left.+(E-M)(\boldsymbol{k}-\boldsymbol{q})^{2}\right\} \\
E= & \sqrt{M^{2}+(\boldsymbol{k}+\boldsymbol{q})^{2}}, \\
\omega_{1}= & \sqrt{m_{1}^{2}+\boldsymbol{k}^{2}}, \quad \omega_{2}=\sqrt{m_{2}^{2}+\boldsymbol{q}^{2}}
\end{aligned}
$$

In these expressions, $M$ and $m_{i}$ are the masses of a baryon and the mesons. The more complicated integrand in $\left|t^{(8 a)}\right|^{2}$ arises because of the $\bar{u} \gamma^{\mu}(k-q)_{\mu} u$ factor when one derivative is included as in Eq. (10). We neglect the negative-energy intermediate baryon propagator as this is suppressed by a further power of $q / M$, leading only to a small relativistic correction. The $k^{0}$ and $q^{0}$ integrations of Eq. (23) are easily 
carried out, and we obtain

$$
\begin{aligned}
I^{(j)}\left(p^{0} ; B, m_{1}, m_{2}\right)= & \int \frac{d^{3} k}{(2 \pi)^{3}} \int \frac{d^{3} q}{(2 \pi)^{3}}\left|t^{(j)}\right|^{2} \frac{1}{2 \omega_{1}} \frac{1}{2 \omega_{2}} \\
& \times \frac{M}{E} \frac{1}{p^{0}-\omega_{1}-\omega_{2}-E+i \epsilon} .
\end{aligned}
$$

The real part of this integral is divergent. We regularize it with a cutoff $\Lambda$ in the three momenta on $\boldsymbol{k}$ and $\boldsymbol{q}$, which is a parameter of the calculation and its value must be somewhat larger than the scale of the typical pion momenta. On the other hand, we use low-energy Lagrangians with one or two derivatives at most, and thus the cutoff should not be too large; otherwise, terms with more derivatives could become relevant. In this work, we will take $\Lambda$ in the range $700-800 \mathrm{MeV}$, roughly the order of magnitude of the cutoff used to regularize the mesonbaryon loops in the study of the $\bar{K} N$ interaction [43]. With the $\mathcal{L}^{\chi}$ of Sec. II F, the cutoff is smaller in order to reproduce analogous results to those with the $8 s$ Lagrangian.

The imaginary part of the diagram represents the decay width, in accordance with the optical theorem. The total decay width of a member of the antidecuplet to any $B M M$ state is given by

$$
\Gamma_{P}^{(j)}\left(p^{0}\right)=-2 \operatorname{Im} \Sigma_{P}^{(j)}\left(p^{0}\right),
$$

while the partial decay width to a particular channel is given by

$$
\begin{aligned}
\Gamma_{P}^{(j)}\left(p^{0} ; B, m_{1}, m_{2}\right)= & -2 \operatorname{Im}\left(F^{(j)} C_{P, B, m_{1}, m_{2}}^{(j)}\right) \\
& \times I^{(j)}\left(p^{0} ; B, m_{1}, m_{2}\right)\left(F^{(j)} C_{P, B, m_{1}, m_{2}}^{(j)}\right) .
\end{aligned}
$$

As an example, let us give in detail the contribution from $\mathcal{L}^{8 a}$ to the $\Theta_{\overline{10}}$ self-energy

$$
\begin{aligned}
\Sigma_{\Theta}^{8 a}\left(p^{0}\right)= & \left(F^{8 a}\right)^{2}\left[18 I^{8 a}\left(p^{0} ; N, K, \pi\right)\right. \\
& \left.+18 I^{8 a}\left(p^{0} ; N, K, \eta\right)\right],
\end{aligned}
$$

and the contribution from $\mathcal{L}^{8 s}$ to the $\Xi_{\overline{10}}$ self-energy

$$
\begin{aligned}
\Sigma_{\Xi}^{8 s}\left(p^{0}\right)= & \left(F^{8 s}\right)^{2}\left[9 I^{8 s}\left(p^{0} ; \Sigma, \bar{K}, \pi\right)+I^{8 s}\left(p^{0} ; \Sigma, \bar{K}, \eta\right)\right. \\
& \left.+6 I^{8 s}\left(p^{0} ; \Xi, \bar{K}, K\right)+4 I^{8 s}\left(p^{0} ; \Xi, \pi, \eta\right)\right] .
\end{aligned}
$$

The expression for all cases can be derived from Tables IX-XII in App. B.

In Eq. (21), we gave a contribution to the self-energy from one interaction Lagrangian $\mathcal{L}^{(j)}$. For the total selfenergy, the sum should be taken over the five interactions $(j=8 s, 8 a, 27, \chi$, and $M)$ at each vertex. This means that at each vertex function, we should make the replacement as $\left(F^{(j)} C_{P, B, m_{1}, m_{2}}^{(j)}\left|t^{(j)}\right|\right) \rightarrow\left(\left|\sum_{j} F^{(j)} C_{P, B, m_{1}, m_{2}}^{(j)} t^{(j)}\right|\right)$. We shall, however, not take into account interference between the $8 a$ term and the others because of the $p$-wave nature of the term.

\section{B. Inclusion of the $\rho$ meson}

It is known that $N(1710) \rightarrow N \pi \pi$ ( $p$ wave) occurs through the $N \rho$ decay. To keep close to the experimental information, we shall also assume that the pair of mesons in the $8 a$ case reconstruct a vector meson. Hence, we replace the

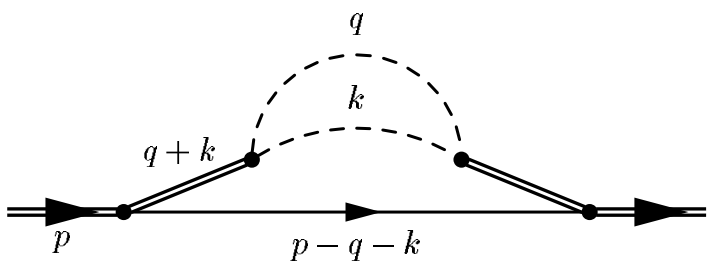

FIG. 2. Self-energy of baryon antidecuplet caused by two-meson cloud with vector meson propagators.

contact interaction of the $\mathcal{L}^{8 a}$ to account for the vector meson propagator (Fig. 2) and include the factor

$$
\frac{m_{v}^{2}}{(q+k)^{2}-m_{v}^{2}},
$$

in each $P \rightarrow B M M$ vertex. The consideration of these contributions needs extra work on the loop integrals since we introduce new poles. The imaginary part of the integrals (associated to placing on-shell the BMM intermediate states) can be easily accounted for by multiplying the integrand of Eq. (23) by

$$
\left|\frac{m_{v}^{2}}{(q+k)^{2}-m_{v}^{2}+i m_{v} \Gamma(q+k)}\right|^{2},
$$

where $\Gamma(q+k)$ accounts for the width of the vector meson ( $\rho$ or $K^{*}$ depending on the $M M$ ) incorporating the energy dependence through the factor $\left(P(q+k) / P_{\text {on }}\right)^{3}$ multiplied to the nominal width, with $P(q+k)$ the relative three momenta of the mesons in the decay of the vector meson in the rest frame and $P_{\mathrm{on}}=P\left(\left(M_{v}, \mathbf{0}\right)\right)$.

For the real part, one must sort out the poles of the vector meson and the intermediate $B M M$ state, which is technically implemented by means of the integral

$$
\begin{aligned}
\operatorname{Re}\{ & \left.I^{8 a}\left(p^{0} ; B, m_{1}, m_{2}\right)\right\} \\
= & -m_{v}^{4} \frac{\partial}{\partial\left(m_{v}^{2}\right)} \mathrm{P} . \mathrm{V} \cdot \int \frac{d^{4} q}{(2 \pi)^{4}} \int \frac{d^{4} k}{(2 \pi)^{4}}\left|t^{8 a}\right|^{2} \\
& \times \frac{1}{k^{2}-m_{1}^{2}+i \epsilon} \frac{1}{q^{2}-m_{2}^{2}+i \epsilon} \\
& \times \frac{1}{(k+q)^{2}-m_{v}^{2}+i \epsilon} \\
& \times \frac{M}{E} \frac{1}{p^{0}-k^{0}-q^{0}-E+i \epsilon}
\end{aligned}
$$

where P.V. stands for the principal value. Here, we neglected the width of the vector meson, which does not play much of a role in the off-shell regions of integrations. The $k^{0}$ and $q^{0}$ integrations can be performed analytically, and one obtains the simple expression

$$
\begin{aligned}
\operatorname{Re} & \left\{I^{8 a}\left(p^{0} ; B, m_{1}, m_{2}\right)\right\} \\
= & m_{v}^{4} \frac{\partial}{\partial\left(m_{v}^{2}\right)} \text { P.V. } \int \frac{d^{3} k}{(2 \pi)^{3}} \int \frac{d^{3} q}{(2 \pi)^{3}}\left|t^{8 a}\right|^{2} \\
& \times \frac{1}{2 \omega_{1}} \frac{1}{2 \omega_{2}} \frac{1}{\omega_{v}} \frac{1}{\omega_{v}+\omega_{1}+\omega_{2}}
\end{aligned}
$$




$$
\begin{aligned}
& \times \frac{1}{p_{0}-\omega_{v}-E+i \epsilon} \\
& \times \frac{1}{p_{0}-\omega_{1}-\omega_{2}-E+i \epsilon} \frac{M}{E} \\
& \times\left(\omega_{1}+\omega_{2}+\omega_{v}-p_{0}+E\right),
\end{aligned}
$$

where $\omega_{v}$ is the on-shell energy of the vector meson.

\section{NUMERICAL EXAMPLES}

Next we present some numerical results that illustrate the antidecuplet mass shifts and decay widths to three-body channels. One of the most exciting aspects in the antidecuplet is that the $\Theta^{+}$is located about $30 \mathrm{MeV}$ below the $N K \pi$ threshold. Hence, it cannot decay into this or any other $B M M$ channel to which it couples. For the interaction Lagrangians, we obtain the $g^{(j)}$ coefficients from the experimentally allowed decay amplitudes of the $N(1710)$. We give several examples that illustrate the general behavior of the two-meson cloud, common to the Lagrangians described in previous sections.

Before studying each of the Lagrangians, let us recall that the mass splitting of the antidecuplet has a contribution which follows the GMO rule, and it would be originated by the difference of the masses of the constituent quarks and their correlations. To this, we add the splitting coming from the real part of the self-energy due to the meson cloud that we are studying. Thus, the masses of the antidecuplet are approximately given by

$$
\begin{aligned}
& M_{\Theta_{\overline{10}}}=M_{0}+\operatorname{Re} \Sigma_{\Theta_{\overline{10}}}, \\
& M_{N_{\overline{10}}}=M_{0}+\operatorname{Re} \Sigma_{N_{\overline{10}}}+\Delta, \\
& M_{\Sigma_{\overline{10}}}=M_{0}+\operatorname{Re} \Sigma_{\Sigma_{\overline{10}}}+2 \Delta, \\
& M_{\Xi_{\overline{10}}}=M_{0}+\operatorname{Re} \Sigma_{\Xi_{\overline{10}}}+3 \Delta,
\end{aligned}
$$

where $M_{0}$ is the bare mass of the antidecuplet and $\Delta$ is the GMO mass splitting, part of which simply comes from the difference of the constituent quark masses. In the constituent quark model, $\Delta$ is related to the difference between the constituent masses of $u, d$, and $s$ quarks, $3 \Delta=\left\langle m_{s}-\right.$ $\left.m_{u, d}\right\rangle_{\text {baryon. }}$. Certainly, quark correlations can also contribute to the experimental value of $\Delta$.

The difference between the light and strange quark masses has been obtained, for example, from hyperfine splittings, in Ref. [44],

$$
\begin{aligned}
\left\langle m_{s}-m_{u}\right\rangle_{\text {meson }} & =\frac{3\left(M_{K^{*}}-M_{\rho}\right)+\left(M_{K}-M_{\pi}\right)}{4} \\
& \simeq 180 \mathrm{MeV},
\end{aligned}
$$

whereas for baryons,

$$
\begin{aligned}
\left\langle m_{s}-m_{u}\right\rangle_{\text {baryon }} & =M_{\Lambda}-M_{N} \simeq 177 \mathrm{MeV}, \\
\left\langle m_{s}-m_{u}\right\rangle_{\text {baryon }} & =\frac{M_{N}+M_{\Delta}}{6}\left(\frac{M_{\Delta}-M_{N}}{M_{\Sigma^{*}}-M_{\Sigma}}-1\right) \\
& \simeq 190 \mathrm{MeV} .
\end{aligned}
$$

But other differences like $M_{K^{*}}-M_{\rho}, M_{\Xi}-M_{N}$, or $M_{\Sigma}-$ $M_{N}$ suggest a wider range, from 122 to 190 and $250 \mathrm{MeV}$, respectively. As we will see, the values of $3 \Delta$ needed within

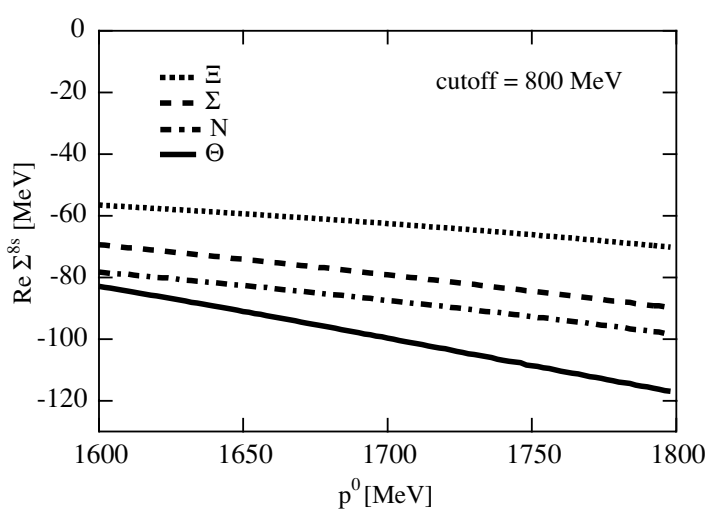

FIG. 3. Mass shifts of baryon antidecuplet $\left(\operatorname{Re} \Sigma_{P}\right)$ due to twomeson cloud from $\mathcal{L}^{8 s}$ with cutoff $=800 \mathrm{MeV} ; p^{0}$ dependence.

this work are of this order of magnitude but somewhat larger, leaving room for extra quark correlation effects.

\section{A. Antidecuplet mass shift with $\mathcal{L}^{8 s}$ and $\mathcal{L}^{8 a}$}

To fix the couplings of the Lagrangians, we start by taking $\mathcal{L}^{8 s}$ and $\mathcal{L}^{8 a}$ defined above and adjusting the coupling constants to obtain the partial decay widths of the $N(1710)$ to $N \pi \pi$ ( $s$ wave, isoscalar) and $N \rho \rightarrow N \pi \pi$ ( $p$ wave, isovector), respectively. These are controlled by the imaginary part of the self-energies (29), which are finite and independent of the cutoff. The central values in the Particle Data Group (PDG) [28] are

$$
\begin{aligned}
\Gamma(N \pi \pi, s \text { wave }) & =25 \mathrm{MeV} \\
\Gamma(N \pi \pi, p \text { wave }) & =15 \mathrm{MeV},
\end{aligned}
$$

and the uncertainties (counting those of the branching ratio and the total width) can be a large fraction of these numbers.

A fit to these central values gives us

$$
g^{8 s}=1.9, \quad g^{8 a}=0.32 .
$$

With these couplings, we calculate the real part of the selfenergies for all the antidecuplet. For the bare antidecuplet mass $p^{0}$ as input, we take an average value of $p^{0}=1700 \mathrm{MeV}$. We also performed a calculation with different values of $p^{0}$ and found that the results have the same qualitative trend, but the depth of the binding varies. To estimate the binding, we show the mass shift from the $\mathcal{L}^{8 s}$ with respect to $p^{0}$ in Fig. 3. We see that, independently of the values of $p^{0}$, all the self-energies are attractive, and that the interaction is more attractive the larger the strangeness; hence, the $\Theta_{\overline{10}}$ is always more bound.

In Fig. 4 we show the results for the contributions from $\mathcal{L}^{8 s}$ and total contributions of $\mathcal{L}^{8 a}$ and $\mathcal{L}^{8 s}$, with $p^{0}=1700 \mathrm{MeV}$ and cutoffs of 700 and $800 \mathrm{MeV}$. The numerical values of the mass shifts are displayed in Table I. We see that $\mathcal{L}^{8 s}$ provides more binding than $\mathcal{L}^{8 a}$ for the same cutoff. The total binding for $\Theta_{\overline{10}}$ ranges from 90 to about $130 \mathrm{MeV}$, depending on the cutoff. The splitting between the $\Theta_{\overline{10}}$ and $\Xi_{\overline{10}}$ states is about $45 \mathrm{MeV}$ for a cutoff of $700 \mathrm{MeV}$ and $60 \mathrm{MeV}$ for a cutoff of $800 \mathrm{MeV}$. Since the experimental splitting is $320 \mathrm{MeV}$ for the 

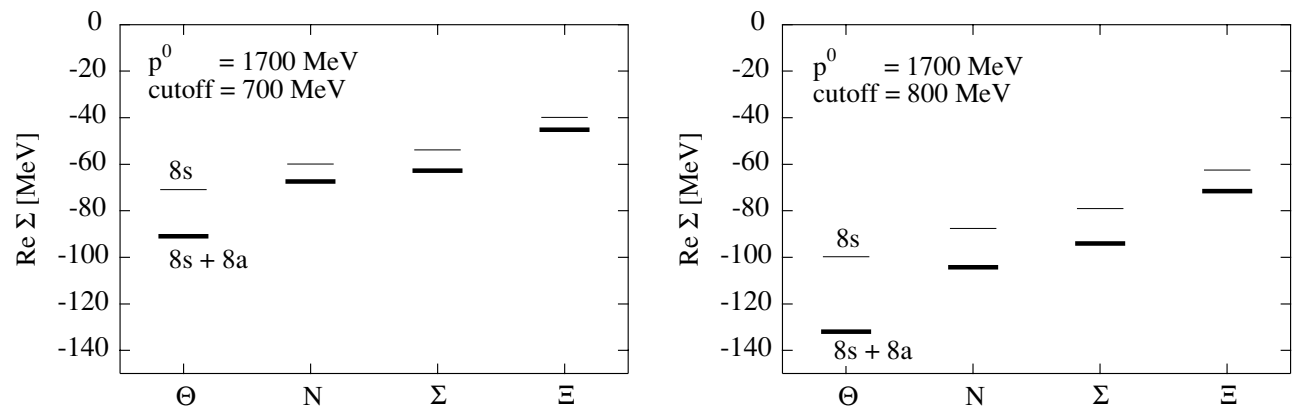

FIG. 4. Mass shifts of baryon antidecuplet $\left(\operatorname{Re} \Sigma_{P}\right)$ due to two-meson cloud with $p^{0}=1700 \mathrm{MeV}$ at two cutoff values. Thin lines represent the results from contributions from $\mathcal{L}^{8 s}$, and thick lines denote the total contribution with $\mathcal{L}^{8 s}$ and $\mathcal{L}^{8 a}$.

$\Theta(1540)$ and $\Xi(1860)$, the splitting provided by the two-meson cloud is on the order of $20 \%$ of the experimental one.

We believe these magnitudes to be realistic (and hence one of the reasons to settle for a cutoff) based on the findings of Ref. [22] that the meson-baryon interaction is insufficient to bind the $K \pi N$ system and that one has to increase the interaction by about a factor of 5 to have the three-particle system bound. Indeed, had the nature of the $\Theta(1540)$ been that of the $K \pi N$ system, we would have obtained all the splitting from the two-meson cloud. There is, hence, a qualitative correlation between the moderate amount of the two-meson cloud contribution claimed here and the difficulty to make the stable $K \pi N$ system based solely on the $K \pi N$ dynamics.

Next we present the antidecuplet spectrum generated with the splitting obtained here. We take the cutoff of $800 \mathrm{MeV}$ for reference. Inserting $M_{\Theta_{\overline{10}}}=1540 \mathrm{MeV}$ and $M_{\Xi_{\overline{10}}}=$ $1860 \mathrm{MeV}$ in Eq. (36), together with our calculated selfenergies, we obtain $M_{0}=1670 \mathrm{MeV}$ and $\Delta=87.5 \mathrm{MeV}$, then

$$
\begin{aligned}
& M_{\Theta_{\overline{10}}}=1540 \mathrm{MeV} \quad \text { (input), } \\
& M_{N_{\overline{10}}}=1652 \mathrm{MeV}, \\
& M_{\Sigma_{\overline{10}}}=1749 \mathrm{MeV}, \\
& M_{\Xi_{\overline{10}}}=1860 \mathrm{MeV} \quad \text { (input). }
\end{aligned}
$$

The value $3 \Delta \sim 260 \mathrm{MeV}$ is fairly reasonable for our estimate purposes. It would indicate, however, that about $30 \mathrm{MeV}$ of $\Delta$, above the $60 \mathrm{MeV}$ coming from the constituent quark consideration, would come from quark correlations. The large $\Theta_{\overline{10}}$ binding with respect to that of the $N_{\overline{10}}$ state is responsible

TABLE I. Mass shifts of baryon antidecuplet $\left(\operatorname{Re} \Sigma_{P}\right)$ due to twomeson cloud with $p^{0}=1700 \mathrm{MeV}$ and cutoffs 700 and $800 \mathrm{MeV}$. All values are shown in units of $\mathrm{MeV}$.

\begin{tabular}{lrrrrrrrr}
\hline \hline & \multicolumn{3}{c}{ Cutoff $=700 \mathrm{MeV}$} & & \multicolumn{3}{c}{ Cutoff $=800 \mathrm{MeV}$} \\
\cline { 2 - 3 } \cline { 7 - 8 } & $\mathcal{L}^{8 s}$ & $\mathcal{L}^{8 a}$ & Total & & $\mathcal{L}^{8 s}$ & $\mathcal{L}^{8 a}$ & Total \\
\hline$\Theta_{\overline{10}}$ & -71 & -20 & -91 & & -100 & -32 & -132 \\
$N_{\overline{10}}$ & -60 & -7 & -67 & & -87 & -17 & -104 \\
$\Sigma_{\overline{10}}$ & -54 & -9 & -63 & & -79 & -15 & -94 \\
$\Xi_{\overline{10}}$ & -40 & -5 & -45 & & -63 & -9 & -72 \\
\hline \hline
\end{tabular}

for the new value $M_{N_{\overline{10}}}=1652 \mathrm{MeV}$, which is slightly higher than the value we would obtain from an exact GMO rule splitting $(1646 \mathrm{MeV})$, but still far from the $1710 \mathrm{MeV}$ resonance we have assumed for the antidecuplet. As discussed in the introduction, a necessary mixture of an octet representation with the antidecuplet could bring the mass close to that of the $N(1710)$, although the possibility of having a new $N^{*}$ resonance belonging to the antidecuplet cannot be ruled out [45].

\section{B. Antidecuplet decay widths from $\mathcal{L}^{8 s}$ and $\mathcal{L}^{8 a}$}

Now we show the partial decay widths obtained according to Eq. (29). As already mentioned, $\Theta(1540)$ has no $B M M$ channel to decay. Among all decay channels, the $N(1710)$ decays broadly into $N \pi \pi$, and it can also decay into $N \pi \eta$. The $\Sigma(1770)$ can decay into $N \bar{K} \pi, \Lambda \pi \pi$, and $\Sigma \pi \pi$, and the $\Xi(1860)$ into $\Sigma \bar{K} \pi$ and $\Xi \pi \pi$, because of the threshold energies of $B M M$ channels.

To calculate the decay, since the phase space is essential for the imaginary part, we take the observed masses,

$$
M_{N_{\overline{10}}}=1710, \quad M_{\Sigma_{\overline{10}}}=1770, \quad M_{\Xi_{\overline{10}}}=1860 .
$$

The results appear in Table II. We can see that the widths are not very large for all channels. Among them, we obtain the partial decay widths of the $\Sigma(1770)$ into $\Sigma \pi \pi$ and $N \bar{K} \pi$. When compared with the experimental data, indeed, the $\Sigma(1770)$ would have a total width into two-meson and baryon of about $24 \mathrm{MeV}$, which is well within the total width of the $\Sigma(1770)$ of

TABLE II. Partial decay widths for the allowed channels and total width for any $B M M$ channel, at the masses of the antidecuplet members. All values are in $\mathrm{MeV}$.

\begin{tabular}{lccc}
\hline \hline Decay widths $(\mathrm{MeV})$ & $\Gamma^{(8 s)}$ & $\Gamma^{(8 a)}$ & $\Gamma_{B M M}^{\text {tot }}$ \\
\hline$N(1710) \rightarrow N \pi \pi$ (inputs) & 25 & 15 & 40 \\
$N(1710) \rightarrow N \eta \pi$ & 0.58 & - & \\
$\Sigma(1770) \rightarrow N \bar{K} \pi$ & 4.7 & 6.0 & 24 \\
$\Sigma(1770) \rightarrow \Sigma \pi \pi$ & 10 & 0.62 & \\
$\Sigma(1770) \rightarrow \Lambda \pi \pi$ & - & 2.9 & \\
$\Xi(1860) \rightarrow \Sigma \bar{K} \pi$ & 0.57 & 0.46 & 2.1 \\
$\Xi(1860) \rightarrow \Xi \pi \pi$ & - & 1.1 & \\
\hline \hline
\end{tabular}


TABLE III. Mass shifts of baryon antidecuplet $\left(\operatorname{Re} \Sigma_{P}\right)$ due to two-meson cloud with $800 \mathrm{MeV}$ cutoff for $\mathcal{L}^{8 s}$ and $525 \mathrm{MeV}$ for $\mathcal{L}^{x}$. All values are in $\mathrm{MeV}$.

\begin{tabular}{lcc}
\hline \hline Mass shifts $(\mathrm{MeV})$ & $\mathcal{L}^{8 s}$ & $\mathcal{L}^{x}$ \\
\hline$\Theta_{\overline{10}}$ & -100 & -99 \\
$N_{\overline{10}}$ & -87 & -83 \\
$\Sigma_{\overline{10}}$ & -79 & -70 \\
$\Xi_{\overline{10}}$ & -63 & -57 \\
\hline \hline
\end{tabular}

about $70 \mathrm{MeV}$ [28]. As for the $\Xi(1860)$ resonance, we obtain a total width of about $2 \mathrm{MeV}$, which is certainly compatible with the total width of less smaller than $18 \mathrm{MeV}$ claimed by the NA49 Collaboration [6]. Detailed information of the partial decay widths of these resonances to three-body channels will give us more understanding of the $P B M M$ interaction.

\section{Mass shifts and decay widths from $\mathcal{L}^{x}$}

Here we show results for the interaction Lagrangian given in Sec. II F, namely the two-meson coupling derived from the chiral symmetric Lagrangian $\mathcal{L}^{\chi}$. We fix the coupling constant $g^{\chi}$ from the $N(1710)$ decay to $N \pi \pi$ ( $s$ wave, isoscalar), and we find $g^{\chi}=0.218$. Then the antidecuplet mass shifts and decay widths are calculated. However, for the mass shifts, we obtain binding energies that are too large - on the order of several hundred MeV with cutoffs around 700-800 MeV-because the loop integral is more divergent than the previous $\mathcal{L}^{8 s}$ case. To reach more reasonable results, we decrease the cutoff and find that $\Lambda=525 \mathrm{MeV}$ would give mass shifts similar to those of $\mathcal{L}^{8 s}$ without derivatives.

We compare the mass shifts of $\mathcal{L}^{\chi}$ with cutoff $525 \mathrm{MeV}$ and $\mathcal{L}^{8 s}$ with $800 \mathrm{MeV}$ in Table III. The decay widths obtained from these Lagrangians are given in Table IV. As expected from the fact that the $C^{(j)}$ coefficients of two Lagrangians are identical, we obtain almost the same mass shifts for $\mathcal{L}^{\chi}$ and $\mathcal{L}^{8 s}$ by properly adjusting the cutoffs. The decay widths are considered to be in fair agreement qualitatively, when considering that the values span two orders of magnitude. Some quantitative differences would come from the SU(3) breaking in the meson momenta appearing in the $\mathcal{L}^{\chi}$ loop, and they are regarded as the uncertainty in our analysis.

\section{Effects of $\mathcal{L}^{27}$ and $\mathcal{L}^{M}$}

Next we draw our attention to the $\mathcal{L}^{27}$ and $\mathcal{L}^{M}$ Lagrangians, that we have not yet used. First note that it is unrealistic to

TABLE IV. Partial decay widths for the allowed channels with $\mathcal{L}^{8 s}$ and $\mathcal{L}^{\chi}$, at the masses of the antidecuplet members. All values are in $\mathrm{MeV}$.

\begin{tabular}{lcc}
\hline \hline Decay widths $(\mathrm{MeV})$ & $\Gamma^{(8 s)}$ & $\Gamma^{(\chi)}$ \\
\hline$N(1710) \rightarrow N \pi \pi$ (input) & 25 & 25 \\
$N(1710) \rightarrow N \eta \pi$ & 0.58 & 0.32 \\
$\Sigma(1770) \rightarrow N \bar{K} \pi$ & 4.7 & 4.5 \\
$\Sigma(1770) \rightarrow \Sigma \pi \pi$ & 10 & 3.6 \\
$\Xi(1860) \rightarrow \Sigma \bar{K} \pi$ & 0.57 & 0.40 \\
\hline \hline
\end{tabular}

make these Lagrangians solely responsible for the $N(1710)$ decay width into $N \pi \pi$ ( $s$ wave) channel. This would lead to some unphysical results such as very large decay widths of the $\Xi_{\overline{10}}$ into $B M M$ channels or a large binding energy of several hundred MeV. Hence, combined with the analyses in the previous section, this fact would justify the approach followed in Ref. [26], where only the $\mathcal{L}^{8 s}$ and $\mathcal{L}^{8 a}$ terms are taken to study the $\Theta^{+}$self-energies in a nuclear medium. Thus, assuming that one cannot have a large fraction of these Lagrangians, we will determine to what extent we can allow contributions from $\mathcal{L}^{27}$ and $\mathcal{L}^{M}$.

We first pursue a model that mixes $\mathcal{L}^{8 s}$ and $\mathcal{L}^{27}$. The coupling constants should be determined such that the decay width of $N_{\overline{10}} \rightarrow N \pi \pi$ ( $s$ wave) is unchanged. According to Table VI, the $C_{B, m_{1}, m_{2}}^{(j)}$ coefficients for $N_{\overline{10}} \rightarrow N \pi \pi$ channels are

$$
\begin{aligned}
C_{p \pi^{0} \pi^{0}}^{8 s} & =\frac{1}{\sqrt{2}}, \quad C_{p \pi^{0} \pi^{0}}^{27}=-\frac{2 \sqrt{2}}{5}, \\
C_{p \pi^{+} \pi^{-}}^{8 s} & =\sqrt{2}, \quad C_{p \pi^{+} \pi^{-}}^{27}=-\frac{4 \sqrt{2}}{5} .
\end{aligned}
$$

To see the contribution from each Lagrangian clearly, we set $g^{8 s}=g^{27}=1.88$, and take the combination

$$
a \mathcal{L}^{8 s}+b \mathcal{L}^{27}, \quad b=-\frac{5}{4}(1-a) .
$$

In this case,

$$
\begin{aligned}
& C_{p \pi^{0} \pi^{0}}^{8 s+27}=\frac{1}{\sqrt{2}} a-\frac{2 \sqrt{2}}{5} \times\left(-\frac{5}{4}(1-a)\right)=\frac{1}{\sqrt{2}}, \\
& C_{p \pi^{+} \pi^{-}}^{8 s+27}=\sqrt{2} a-\frac{4 \sqrt{2}}{5} \times\left(-\frac{5}{4}(1-a)\right)=\sqrt{2},
\end{aligned}
$$

and, therefore, we have the same $N(1710) \rightarrow N \pi \pi(s$ wave) decay independent of $a$, but different decays into other channels. With this parametrization, $a=1$ corresponds to the limit where $\mathcal{L}^{27}$ is switched off, while $a=0$ relates to the $\mathcal{L}^{27}$ contribution only. We vary $a$ around 1 and find that for $0.90<a<1.06$, the self-energy results are acceptable on physical grounds. If we exceed this range, the splitting of the different strangeness states of the antidecuplet spoils agreement with the GMO rule. Taking this range of acceptable values of $a$ into account, we find the results for the binding energies shown in Fig. 5. As we see in the figure, $\mathcal{L}^{27}$ tends to contribute to make the binding energy deeper. A possible contribution from $\mathcal{L}^{27}$ would be considered as a theoretical uncertainty in our analysis.

Next we address the $\mathcal{L}^{M}$ term. Once again, as in the $\mathcal{L}^{27}$ case, we set $g^{8 s}=g^{M}=1.88$ and take the combination

$$
a \mathcal{L}^{8 s}+b \mathcal{L}^{M}, \quad b=\frac{f^{2}}{m_{\pi}^{2}}(1-a),
$$

in order to have the same $N(1710) \rightarrow N \pi \pi(s$ wave). In this case, we also see that the values of $0.76<a<1.06$ are acceptable on physical grounds, but larger deviations of $a$ again lead to undesired signs of the splitting between members of the antidecuplet, as well as to unacceptably large results of the binding energies. Within this interval of coupling 

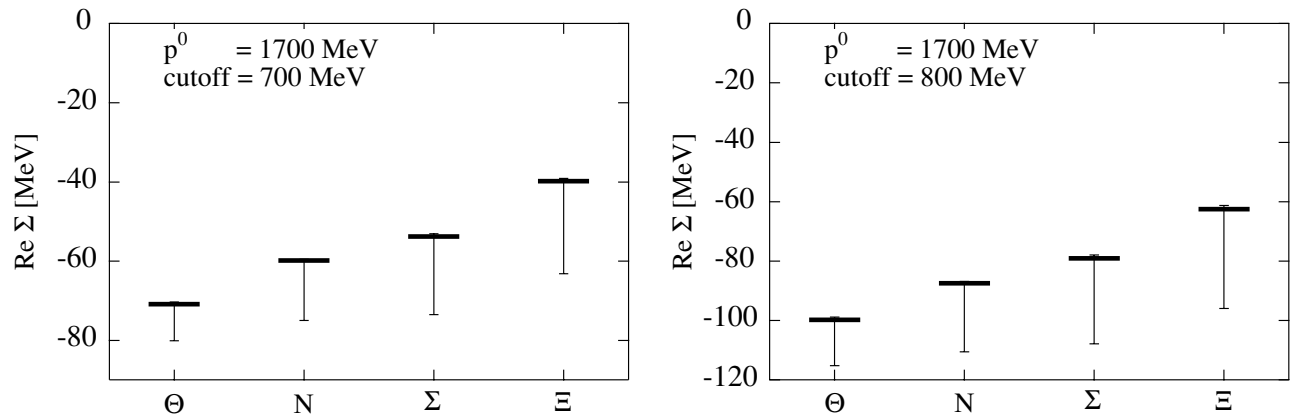

FIG. 5. Mass shifts of baryon antidecuplet due to two-meson cloud from $\mathcal{L}^{8 s}$ and possible $\mathcal{L}^{27}$ contributions at two cutoff values. Horizontal bar: results with $\mathcal{L}^{8 s}$ only. Vertical bar: band of values including $\mathcal{L}^{27}$ in the range of the text.

constant, the results obtained for the binding energies of the antidecuplet members are given in Fig. 6. We observe that $\mathcal{L}^{M}$ also contributes to attractive binding energy, and the splitting of $\Theta_{\overline{10}}$ and $N_{\overline{10}}$ becomes large compared with the other splittings.

\section{DISCUSSION AND CONCLUSION}

The assumptions made throughout the paper and the uncertainties in the experimental input make the nature of our analysis qualitative. We assume that the $\Theta^{+}$is a $1 / 2^{+}$ state with $I=0$ and that it belongs to an antidecuplet. In addition to these minimal assumptions, we consider that the $N(1710)$ also belongs to this same antidecuplet. The meson cloud mechanism proposed in this work leads, in all the cases studied, to the following conclusions:

1. The two-meson cloud yields an attractive self-energy for all members of the antidecuplet. The observation of attraction is consistent with the previous attempts to describe the $\Theta^{+}$ as a $K \pi N$ state [18-20,22].

2. It also contributes to the splitting between antidecuplet members, which is only moderately cutoff dependent and provides about $20 \%$ of the total splitting to a stronger effect for reasonable values of the cutoff. The role played by the two-meson cloud is therefore of relevance for a precise understanding of the nature of the $\Theta^{+}$and the antidecuplet.

3. The magnitude of $20 \%$ is also in agreement quantitatively with the strength of attraction found in the previous study of the BMM three-body system [22]. The values of the mass splitting are such that they still leave some room for quark correlation effects after the GMO mass splitting coming from the mass difference between $u, d$, and $s$ constituent quarks is considered. The contribution to the splitting from the meson cloud is on the same order of magnitude as the one provided by these quark correlations.

4. From the experimental point of view, it is clear that the investigation of the decay channels into two mesons and a baryon of the resonances $N(1710), \Sigma(1770)$, and $\Xi(1860)$ deserves renewed interest.

\section{ACKNOWLEDGMENTS}

This work is supported by the Japan-Europe (Spain) Research Cooperation Program of Japan Society for the Promotion of Science (JSPS) and Spanish Council for Scientific Research (CSIC), which enabled E.O. and M.J.V.V. to visit RCNP, Osaka. This work is partly supported by DGICYT Contract Numbers BFM2003-00856, FPA 2000-0956, and BFM 2002-01003 and the E.U. EURIDICE Network Contract No. HPRN-CT-2002-00311.

\section{APPENDIX A: FLAVOR COEFFICIENTS FOR PBMM VERTICES}

This appendix contains the flavor coefficients for the tree-level vertices in the three Lagrangians considered. The coefficients for $\Theta_{\overline{10}}, N_{\overline{10}}, \Sigma_{\overline{10}}$, and $\Xi_{\overline{10}}$ are shown in Tables $\mathrm{V}$, VI, VII, and VIII, respectively.
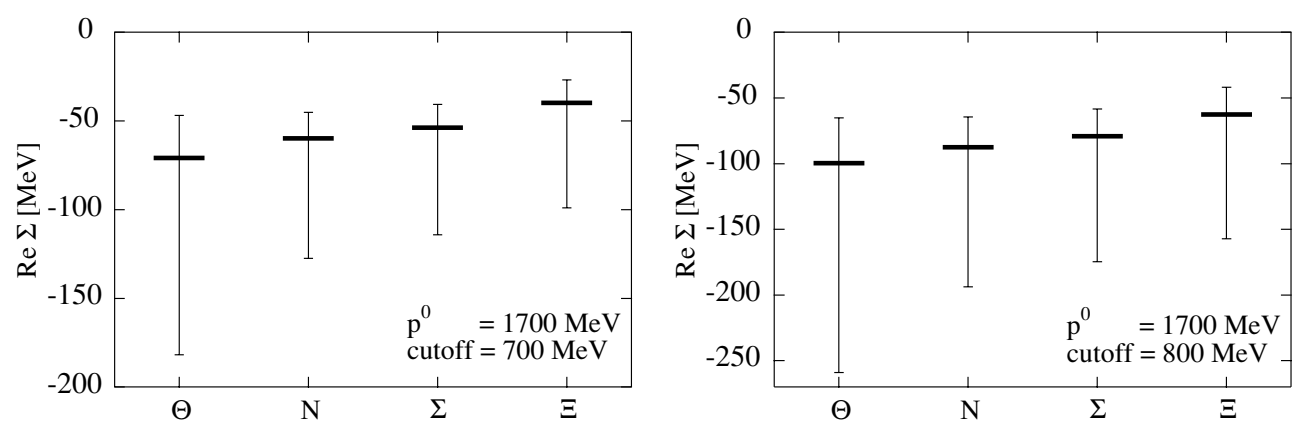

FIG. 6. Mass shifts of baryon antidecuplet due to two-meson cloud from $\mathcal{L}^{8 s}$ and possible $\mathcal{L}^{M}$ contributions at two cutoff values. Horizontal bar: results with $\mathcal{L}^{8 s}$ only. Vertical bar: band of values including $\mathcal{L}^{M}$ in the range of the text. 
TABLE V. The $C_{B, m_{1}, m_{2}}^{(j)}$ flavor coefficients for the vertex with $\Theta_{10}^{+}$, octet baryons, and two octet mesons.

\begin{tabular}{cccccc}
\hline \hline$P$ & $B M M$ & $8 s$ & $8 a$ & 27 & $M$ \\
\hline$\Theta_{10}^{+}$ & $p K^{+} \pi^{-}$ & $-\sqrt{6}$ & $-\sqrt{6}$ & $\frac{4 \sqrt{6}}{5}$ & $-\sqrt{6} \frac{m_{K}^{2}+m_{\pi}^{2}}{2 f^{2}}$ \\
& $p K^{0} \pi^{0}$ & $\sqrt{3}$ & $\sqrt{3}$ & $-\frac{4 \sqrt{3}}{5}$ & $\sqrt{3} \frac{m_{K}^{2}+m_{\pi}^{2}}{2 f^{2}}$ \\
& $p K^{0} \eta$ & 1 & -3 & $\frac{36}{5}$ & $\frac{5 m_{K}^{2}-3 m_{\pi}^{2}}{2 f^{2}}$ \\
& $n K^{+} \pi^{0}$ & $\sqrt{3}$ & $\sqrt{3}$ & $-\frac{4 \sqrt{3}}{5}$ & $\sqrt{3} \frac{m_{K}^{2}+m_{\pi}^{2}}{2 f^{2}}$ \\
$n K^{+} \eta$ & -1 & 3 & $-\frac{36}{5}$ & $-\frac{5 m_{K}^{2}-3 m_{\pi}^{2}}{2 f^{2}}$ \\
$n K^{0} \pi^{+}$ & $\sqrt{6}$ & $\sqrt{6}$ & $-\frac{4 \sqrt{6}}{5}$ & $\sqrt{6} \frac{m_{K}^{2}+m_{\pi}^{2}}{2 f^{2}}$ \\
$\Sigma^{+} K^{0} K^{0}$ & - & - & $-4 \sqrt{6}$ & - \\
$\Sigma^{0} K^{0} K^{+}$ & - & - & $-8 \sqrt{3}$ & - \\
$\Sigma^{-} K^{+} K^{+}$ & - & - & $4 \sqrt{6}$ & - \\
\hline \hline
\end{tabular}

TABLE VI. The $C_{B, m_{1}, m_{2}}^{(j)}$ flavor coefficients for the vertex with $N_{\overline{10}}^{+}$, octet baryons, and two octet mesons. Coefficients for $N_{10}^{0}$ are obtained by using isospin symmetry.

\begin{tabular}{|c|c|c|c|c|c|}
\hline$P$ & $B M M$ & $8 s$ & $8 a$ & 27 & $M$ \\
\hline \multirow[t]{21}{*}{$N_{\overline{10}}^{+}$} & $p K^{+} K^{-}$ & $-\sqrt{2}$ & $-\sqrt{2}$ & $\frac{4 \sqrt{2}}{5}$ & $-\sqrt{2} \frac{m_{K}^{2}}{f^{2}}$ \\
\hline & $p K^{0} \bar{K}^{0}$ & - & $-2 \sqrt{2}$ & $4 \sqrt{2}$ & - \\
\hline & $p \pi^{0} \pi^{0}$ & $\frac{1}{\sqrt{2}}$ & - & $-\frac{2 \sqrt{2}}{5}$ & $\frac{1}{\sqrt{2}} \frac{m_{\pi}^{2}}{f^{2}}$ \\
\hline & $p \pi^{+} \pi^{-}$ & $\sqrt{2}$ & $\sqrt{2}$ & $-\frac{4 \sqrt{2}}{5}$ & $\sqrt{2} \frac{m_{\pi}^{2}}{f^{2}}$ \\
\hline & $p \eta \eta$ & $-\frac{1}{\sqrt{2}}$ & - & $-\frac{18 \sqrt{2}}{5}$ & $-\frac{1}{\sqrt{2}} \frac{8 m_{K}^{2}-5 m_{\pi}^{2}}{3 f^{2}}$ \\
\hline & $p \eta \pi^{0}$ & $-\sqrt{\frac{2}{3}}$ & - & $\frac{8 \sqrt{6}}{5}$ & $-\sqrt{\frac{2}{3}} \frac{m_{\pi}^{2}}{f^{2}}$ \\
\hline & $n \bar{K}^{0} K^{+}$ & $-\sqrt{2}$ & $-\sqrt{2}$ & $-\frac{16 \sqrt{2}}{5}$ & $-\sqrt{2} \frac{m_{K}^{2}}{f^{2}}$ \\
\hline & $n \pi^{+} \eta$ & $-\frac{2}{\sqrt{3}}$ & - & $\frac{16 \sqrt{3}}{5}$ & $-\frac{2}{\sqrt{3}} \frac{m_{\pi}^{2}}{f^{2}}$ \\
\hline & $n \pi^{+} \pi^{0}$ & - & -2 & - & - \\
\hline & $\Lambda K^{+} \pi^{0}$ & $-\sqrt{\frac{3}{2}}$ & $-\sqrt{\frac{3}{2}}$ & $\frac{2 \sqrt{6}}{5}$ & $-\sqrt{\frac{3}{2}} \frac{m_{K}^{2}+m_{\pi}^{2}}{2 f^{2}}$ \\
\hline & $\Lambda K^{+} \eta$ & $\frac{1}{\sqrt{2}}$ & $-\frac{3}{\sqrt{2}}$ & $\frac{18 \sqrt{2}}{5}$ & $\frac{1}{\sqrt{2}} \frac{5 m_{K}^{2}-3 m_{\pi}^{2}}{2 f^{2}}$ \\
\hline & $\Lambda K^{0} \pi^{+}$ & $-\sqrt{3}$ & $-\sqrt{3}$ & $\frac{4 \sqrt{3}}{5}$ & $-\sqrt{3} \frac{m_{K}^{2}+m_{\pi}^{2}}{2 f^{2}}$ \\
\hline & $\Sigma^{+} K^{+} \pi^{-}$ & $\sqrt{2}$ & $\sqrt{2}$ & $-\frac{4 \sqrt{2}}{5}$ & $\sqrt{2} \frac{m_{K}^{2}+m_{\pi}^{2}}{2 f^{2}}$ \\
\hline & $\Sigma^{+} K^{0} \eta$ & $-\frac{1}{\sqrt{3}}$ & $\sqrt{3}$ & $\frac{28 \sqrt{3}}{5}$ & $-\frac{1}{\sqrt{3}} \frac{5 m_{K}^{2}-3 m_{\pi}^{2}}{2 f^{2}}$ \\
\hline & $\Sigma^{+} K^{0} \pi^{0}$ & -1 & -1 & $-\frac{36}{5}$ & $-\frac{m_{K}^{2}+m_{\pi}^{2}}{2 f^{2}}$ \\
\hline & $\Sigma^{0} K^{+} \pi^{0}$ & $\frac{1}{\sqrt{2}}$ & $\frac{1}{\sqrt{2}}$ & $-\frac{22 \sqrt{2}}{5}$ & $\frac{1}{\sqrt{2}} \frac{m_{K}^{2}+m_{\pi}^{2}}{2 f^{2}}$ \\
\hline & $\Sigma^{0} K^{0} \pi^{+}$ & 1 & 1 & $\frac{36}{5}$ & $\frac{m_{K}^{2}+m_{\pi}^{2}}{2 f^{2}}$ \\
\hline & $\Sigma^{0} K^{+} \eta$ & $-\frac{1}{\sqrt{6}}$ & $\sqrt{\frac{3}{2}}$ & $\frac{14 \sqrt{6}}{5}$ & $-\frac{1}{\sqrt{6}} \frac{5 m_{K}^{2}-3 m_{\pi}^{2}}{2 f^{2}}$ \\
\hline & $\Sigma^{-} K^{+} \pi^{+}$ & - & - & $-8 \sqrt{2}$ & - \\
\hline & $\Xi^{-} K^{+} K^{+}$ & - & - & $4 \sqrt{2}$ & - \\
\hline & $\Xi^{0} K^{+} K^{0}$ & - & - & $4 \sqrt{2}$ & - \\
\hline
\end{tabular}

TABLE VII. The $C_{B, m_{1}, m_{2}}^{(j)}$ flavor coefficients for the vertex with $\Sigma_{\overline{10}}^{+}$, octet baryons, and two octet mesons. Coefficients for $\Sigma_{\overline{10}}^{0}$ and $\Sigma_{\overline{10}}^{-}$are obtained by using isospin symmetry.

\begin{tabular}{|c|c|c|c|c|c|}
\hline$P$ & $B M M$ & $8 s$ & $8 a$ & 27 & $M$ \\
\hline \multirow[t]{21}{*}{$\Sigma_{\frac{+}{10}}^{+}$} & $p \pi^{+} K^{-}$ & $-\sqrt{2}$ & $-\sqrt{2}$ & $\frac{4 \sqrt{2}}{5}$ & $-\sqrt{2} \frac{m_{K}^{2}+m_{\pi}^{2}}{2 f^{2}}$ \\
\hline & $p \pi^{0} \bar{K}^{0}$ & 1 & 1 & $-\frac{24}{5}$ & $\frac{m_{K}^{2}+m_{\pi}^{2}}{2 f^{2}}$ \\
\hline & $p \bar{K}^{0} \eta$ & $\frac{1}{\sqrt{3}}$ & $\sqrt{3}$ & $\frac{32 \sqrt{3}}{5}$ & $\frac{1}{\sqrt{3}} \frac{5 m_{K}^{2}-3 m_{\pi}^{2}}{2 f^{2}}$ \\
\hline & $n \bar{K}^{0} \pi^{+}$ & - & - & $-4 \sqrt{2}$ & - \\
\hline & $\Lambda \bar{K}^{0} K^{+}$ & $-\sqrt{3}$ & $-\sqrt{3}$ & $-\frac{16 \sqrt{3}}{5}$ & $-\sqrt{3} \frac{m_{K}^{2}}{f^{2}}$ \\
\hline & $\Lambda \pi^{+} \eta$ & $-\sqrt{2}$ & - & $\frac{24 \sqrt{2}}{5}$ & $-\sqrt{2} \frac{m_{\pi}^{2}}{f^{2}}$ \\
\hline & $\Lambda \pi^{+} \pi^{0}$ & - & $-\sqrt{6}$ & - & - \\
\hline & $\Sigma^{+} K^{+} K^{-}$ & $-\sqrt{2}$ & $-\sqrt{2}$ & $\frac{4 \sqrt{2}}{5}$ & $-\sqrt{2} \frac{m_{K}^{2}}{f^{2}}$ \\
\hline & $\Sigma^{+} \pi^{+} \pi^{-}$ & $\sqrt{2}$ & $\sqrt{2}$ & $-\frac{4 \sqrt{2}}{5}$ & $\sqrt{2} \frac{m_{\pi}^{2}}{f^{2}}$ \\
\hline & $\Sigma^{+} \eta \eta$ & $-\frac{1}{\sqrt{2}}$ & - & $\frac{12 \sqrt{2}}{5}$ & $-\frac{1}{\sqrt{2}} \frac{8 m_{K}^{2}-5 m_{\pi}^{2}}{3 f^{2}}$ \\
\hline & $\Sigma^{+} \eta \pi^{0}$ & $-\sqrt{\frac{2}{3}}$ & - & $-\frac{12 \sqrt{6}}{5}$ & $-\sqrt{\frac{2}{3}} \frac{m_{\pi}^{2}}{f^{2}}$ \\
\hline & $\Sigma^{+} \pi^{0} \pi^{0}$ & $\frac{1}{\sqrt{2}}$ & - & $\frac{8 \sqrt{2}}{5}$ & $\frac{1}{\sqrt{2}} \frac{m_{\pi}^{2}}{f^{2}}$ \\
\hline & $\Sigma^{+} K^{0} \bar{K}^{0}$ & - & $-2 \sqrt{2}$ & $-4 \sqrt{2}$ & - \\
\hline & $\Sigma^{0} \bar{K}^{0} K^{+}$ & 1 & 1 & $-\frac{24}{5}$ & $\frac{m_{K}^{2}}{f^{2}}$ \\
\hline & $\Sigma^{0} \eta \pi^{+}$ & $\sqrt{\frac{2}{3}}$ & - & $\frac{12 \sqrt{6}}{5}$ & $\sqrt{\frac{2}{3}} \frac{m_{\pi}^{2}}{f^{2}}$ \\
\hline & $\Sigma^{0} \pi^{+} \pi^{0}$ & - & $\sqrt{2}$ & $-4 \sqrt{2}$ & - \\
\hline & $\Sigma^{-} \pi^{+} \pi^{+}$ & - & - & $-4 \sqrt{2}$ & - \\
\hline & $\Xi^{0} K^{+} \pi^{0}$ & 1 & 1 & $-\frac{24}{5}$ & $\frac{m_{K}^{2}+m_{\pi}^{2}}{2 f^{2}}$ \\
\hline & $\Xi^{0} K^{0} \pi^{+}$ & $\sqrt{2}$ & $\sqrt{2}$ & $\frac{16 \sqrt{2}}{5}$ & $\sqrt{2} \frac{m_{K}^{2}+m_{\pi}^{2}}{2 f^{2}}$ \\
\hline & $\Xi^{0} K^{+} \eta$ & $-\frac{1}{\sqrt{3}}$ & $-\sqrt{3}$ & $\frac{8 \sqrt{3}}{5}$ & $-\frac{1}{\sqrt{3}} \frac{5 m_{K}^{2}-3 m_{\pi}^{2}}{2 f^{2}}$ \\
\hline & $\Xi^{-} K^{+} \pi^{+}$ & - & - & $8 \sqrt{2}$ & - \\
\hline
\end{tabular}

TABLE VIII. The $C_{B, m_{1}, m_{2}}^{(j)}$ flavor coefficients for the vertex with $\Xi_{\overline{10}}^{+}$, octet baryons, and two octet mesons. Coefficients for $\Xi_{\overline{10}}^{0}, \Xi_{\overline{10}}^{-}$, and $\Xi_{\overline{10}}^{--}$are obtained by using isospin symmetry.

\begin{tabular}{cccccc}
\hline \hline$P$ & $B M M$ & $8 s$ & $8 a$ & 27 & $M$ \\
\hline$\Xi_{\overline{10}}^{+}$ & $\Sigma^{+} \pi^{+} K^{-}$ & $-\sqrt{6}$ & $-\sqrt{6}$ & $\frac{4 \sqrt{6}}{5}$ & $-\sqrt{6} \frac{m_{K}^{2}+m_{\pi}^{2}}{2 f^{2}}$ \\
& $\Sigma^{+} \pi^{0} \bar{K}^{0}$ & $\sqrt{3}$ & $\sqrt{3}$ & $\frac{16 \sqrt{3}}{5}$ & $\sqrt{3} \frac{m_{K}^{2}+m_{\pi}^{2}}{2 f^{2}}$ \\
$\Sigma^{+} \bar{K}^{0} \eta$ & 1 & 3 & $-\frac{24}{5}$ & $\frac{5 m_{K}^{2}-3 m_{\pi}^{2}}{2 f^{2}}$ \\
$\Sigma^{0} \bar{K}^{0} \pi^{+}$ & - & - & $-4 \sqrt{3}$ & - \\
$\Lambda \bar{K}^{0} \pi^{+}$ & - & - & -12 & - \\
$p \bar{K}^{0} \bar{K}^{0}$ & - & - & $-4 \sqrt{6}$ & - \\
$\Xi^{0} \bar{K}^{0} K^{+}$ & $\sqrt{6}$ & $\sqrt{6}$ & $-\frac{4 \sqrt{6}}{5}$ & $\sqrt{6} \frac{m_{K}^{2}}{f^{2}}$ \\
$\Xi^{0} \eta \pi^{+}$ & 2 & - & $\frac{12}{5}$ & $2 \frac{m_{\pi}^{2}}{f^{2}}$ \\
$\Xi^{0} \pi^{+} \pi^{0}$ & - & $2 \sqrt{3}$ & $-4 \sqrt{3}$ & - \\
$\Xi^{-} \pi^{+} \pi^{+}$ & - & - & $4 \sqrt{6}$ & - \\
\hline \hline
\end{tabular}


TABLE IX. The $D_{\alpha}^{(j)}$ coefficients for the $\Theta_{\overline{10}}$ self-energies.

\begin{tabular}{cccccc}
\hline \hline$P$ & $\alpha$ & $8 s$ & $8 a$ & $8 s+27$ & $8 s+M$ \\
\hline$\Theta_{\overline{10}}$ & $N K \pi$ & 18 & 18 & 18 & $18\left(a+\frac{m_{K}^{2}+m_{\pi}^{2}}{2 f^{2}} b\right)^{2}$ \\
& $N K \eta$ & 2 & 18 & $2\left(a+\frac{36}{5} b\right)^{2}$ & $2\left(a+\frac{5 m_{K}^{2}-3 m_{\pi}^{2}}{2 f^{2}} b\right)^{2}$ \\
& $\Sigma K \pi$ & - & - & $576 b^{2}$ & - \\
\hline \hline
\end{tabular}

\section{APPENDIX B: SELF-ENERGY FORMULAS}

Below are the formulas for calculating the self-energies as described in Eq. (21). In the isospin symmetric limit,

$$
\Sigma_{P}^{(j)}=\left(F^{(j)}\right)^{2} \sum_{\alpha} I^{(j)}(\alpha) D_{P, \alpha}^{(j)}
$$

with $\alpha$ being the $B M M$ channel in the isospin basis, such as $N K \pi, N K \eta$, etc., and $D^{(j)}$ are expressed as the sum of the $\left(C^{(j)}\right)^{2}$. In Tables IX-XII, we show the $D^{(j)}$ coefficients. For the 27 and $M$ cases, following the procedure in Sec. IV D, we set $g^{8 s}=g^{27}=g^{M}=1.88$ and take

$$
a \mathcal{L}^{8 s}+b \mathcal{L}^{27}, \quad b=-\frac{5}{4}(1-a)
$$

and

$$
a \mathcal{L}^{8 s}+b \mathcal{L}^{M}, \quad b=\frac{f^{2}}{m_{\pi}^{2}}(1-a) .
$$

TABLE X. The $D_{\alpha}^{(j)}$ coefficients for the $N_{\overline{10}}$ self-energies.

\begin{tabular}{cccccc}
\hline \hline$P$ & $\alpha$ & $8 s$ & $8 a$ & $8 s+27$ & $8 s+M$ \\
\hline$N_{\overline{10}}$ & $N K \bar{K}$ & 4 & 12 & $2+2\left(a+\frac{16}{5} b\right)^{2}-$ & $4\left(a+\frac{m_{K}^{2}}{f^{2}} b\right)^{2}$ \\
& & & $32 b^{2}$ & $3\left(a+\frac{m_{\pi}^{2}}{f^{2}} b\right)^{2}$ \\
$N \pi \pi$ & 3 & 6 & 3 & $2\left(a+\frac{m_{\pi}^{2}}{f^{2}} b\right)^{2}$ \\
$N \pi \eta$ & 2 & - & $2\left(-a+\frac{24}{5} b\right)^{2}$ & $\left(a+\frac{8 m_{K}^{2}-5 m_{\pi}^{2}}{3 f^{2}} b\right)^{2}$ \\
$N \eta \eta$ & 1 & - & $\left(a+\frac{36}{5}\right)^{2}$ & $\frac{9}{2}\left(a+\frac{m_{K}^{2}+m_{\pi}^{2}}{2 f^{2}} b\right)^{2}$ \\
$\Lambda K \pi$ & $\frac{9}{2}$ & $\frac{9}{2}$ & $\frac{9}{2}$ & $\frac{1}{2}\left(a+\frac{5 m_{K}^{2}-3 m_{\pi}^{2}}{2 f^{2}} b\right)^{2}$ \\
$\Lambda K \eta$ & $\frac{1}{2}$ & $\frac{9}{2}$ & $\frac{1}{2}\left(a+\frac{36}{5}\right)^{2}$ & $\left(a+\frac{m_{K}^{2}+m_{\pi}^{2}}{2 f^{2}} b\right)^{2}$ \\
$\Sigma K \pi$ & $\frac{9}{2}$ & $\frac{9}{2}$ & $2+2\left(a+\frac{36}{5} b\right)^{2}$ & \\
& & & $+\frac{1}{2}\left(a-\frac{44}{5}\right)^{2}+128 b^{2}$ & \\
$\Sigma K \eta$ & $\frac{1}{2}$ & $\frac{9}{2}$ & $\frac{1}{2}\left(-a+\frac{84}{5}\right)^{2}$ & $\frac{1}{2}\left(a+\frac{5 m_{K}^{2}-3 m_{\pi}^{2}}{2 f^{2}} b\right)^{2}$ \\
$\Xi K K$ & - & - & $96 b^{2}$ & - \\
\hline \hline
\end{tabular}

TABLE XI. The $D_{\alpha}^{(j)}$ coefficients for the $\Sigma_{\overline{10}}$ self-energies.

\begin{tabular}{cccccc}
\hline \hline & $\alpha$ & $8 s$ & $8 a$ & $8 s+27$ & $8 s+M$ \\
\hline$\Sigma_{\overline{10}} N \bar{K} \pi$ & 3 & 3 & $2+\left(a-\frac{24}{5} b\right)^{2}+$ & $3\left(a+\frac{m_{K}^{2}+m_{\pi}^{2}}{2 f^{2}} b\right)^{2}$ \\
& $N \bar{K} \eta$ & $\frac{1}{3}$ & 3 & $\frac{1}{3}\left(a+\frac{96}{5} b\right)^{2}$ & $\frac{1}{3}\left(a+\frac{5 m_{K}^{2}-3 m_{\pi}^{2}}{2 f^{2}}\right)^{2}$ \\
$\Lambda K \bar{K}$ & 3 & 3 & $3\left(a+\frac{16}{5} b\right)^{2}$ & $3\left(a+\frac{m_{K}^{2}}{f^{2}} b\right)^{2}$ \\
$\Lambda \pi \eta$ & 2 & - & $2\left(a-\frac{24}{5} b\right)^{2}$ & $2\left(a+\frac{m_{\pi}^{2}}{f^{2}} b\right)^{2}$ \\
$\Lambda \pi \pi$ & - & 6 & - & - \\
$\Sigma K \bar{K}$ & 3 & 11 & $2+\left(a-\frac{24}{5} b\right)^{2}+$ & $3\left(a+\frac{m_{K}^{2}}{f^{2}} b\right)^{2}$ \\
$\Sigma \pi \pi$ & 3 & 4 & $2+\left(a+\frac{16}{5}\right)^{2}+96 b^{2}$ & $\left(a+\frac{m_{\pi}^{2}}{f^{2}} b\right)^{2}$ \\
$\Sigma \pi \eta$ & $\frac{4}{3}$ & - & $\frac{4}{3}\left(a+\frac{36}{5}\right)^{2}$ & $\frac{4}{3}\left(a+\frac{m_{\pi}^{2}}{f^{2}} b\right)^{2}$ \\
$\Sigma \eta \eta$ & 1 & - & $\left(a-\frac{24}{5} b\right)^{2}$ & $\left(a+\frac{8 m_{K}^{2}-5 m_{\pi}^{2}}{3 f^{2}} b\right)^{2}$ \\
$\Xi K \pi$ & 3 & 3 & $2\left(a+\frac{16}{5}\right)^{2}+$ & $3\left(a+\frac{m_{K}^{2}+m_{\pi}^{2}}{2 f^{2}} b\right)^{2}$ \\
$\Xi K \eta$ & $\frac{1}{3}$ & 3 & $\frac{1}{3}\left(a-\frac{24}{5} b\right)^{2}$ & $\frac{1}{3}\left(a+\frac{5 m_{K}^{2}-3 m_{\pi}^{2}}{2 f^{2}} b\right)^{2}$ \\
\hline \hline
\end{tabular}

In these cases, $D^{j}$ are defined as

$$
\Sigma_{P}^{(j)}=\left(F^{(8 s)}\right)^{2} \sum_{\alpha} I^{(j)}(\alpha) D_{P, \alpha}^{(j)},
$$

for $(j)=8 s+27,8 s+M$. One can easily check that when $a=1$ and $b=0$, then $D_{P, \alpha}^{(j)}$ for $(j)=8 s+27,8 s+M$ becomes $D_{P, \alpha}^{(8 s)}$.

TABLE XII. The $D_{\alpha}^{(j)}$ coefficients for the $\Xi_{\overline{10}}$ self-energies.

\begin{tabular}{cccccc}
\hline \hline$P$ & $\alpha$ & $8 s$ & $8 a$ & $8 s+27$ & $8 s+M$ \\
\hline$\Xi_{\overline{10}} \Sigma \bar{K} \pi$ & 9 & 9 & $6+3\left(a+\frac{16}{5} b\right)^{2}+$ & $9\left(a+\frac{m_{K}^{2}+m_{\pi}^{2}}{2 f^{2}} b\right)^{2}$ \\
& & & & $48 b^{2}$ & \\
$\Sigma \bar{K} \eta$ & 1 & 9 & $\left(a-\frac{24}{3} b\right)^{2}$ & $\left(a+\frac{5 m_{K}^{2}-3 m_{\pi}^{2}}{2 f^{2}} b\right)^{2}$ \\
$\Xi K \bar{K}$ & 6 & 6 & 6 & $6\left(a+\frac{m_{K}^{2}}{f^{2}} b\right)^{2}$ \\
$\Xi \pi \eta$ & 4 & - & $4\left(a+\frac{6}{5} b\right)^{2}$ & $4\left(a+\frac{m_{\pi}^{2}}{f^{2}} b\right)^{2}$ \\
$\Xi \pi \pi$ & - & 12 & $240 b^{2}$ & - \\
$\Lambda \bar{K} \pi$ & - & - & $144 b^{2}$ & - \\
$N \bar{K} \bar{K}-$ & - & $192 b^{2}$ & - \\
\hline \hline
\end{tabular}


[1] T. Nakano et al. (LEPS Collaboration), Phys. Rev. Lett. 91, 012002 (2003).

[2] D. Diakonov, V. Petrov, and M. V. Polyakov, Z. Phys. A 359, 305 (1997).

[3] M. Praszalowicz, in Workshop on Skyrmions and Anomalies, edited by M. Jezabek and M. Praszalowicz (World Scientific, Singapore, 1987), p. 112.

[4] http://www.rcnp.osaka-u.ac.jp/ `hyodo/research / Thetapub.html.

[5] International Workshop PENTAQUARK04 at SPring-8, Jul. 2023, 2004, http://www.rcnp.osaka-u.ac.jp/ penta04/.

[6] C. Alt et al. (NA49 Collaboration), Phys. Rev. Lett. 92, 042003 (2004).

[7] H. G. Fischer and S. Wenig, Eur. Phys. J. C 37, 133 (2004).

[8] K. T. Knopfle (HERA-B Collaboration), M. Zavertyaev, and T. Zivko, J. Phys. G 30, S1363 (2004).

[9] WA89 Collaboration, Phys. Rev. C 70, 022201 (2004).

[10] K. Kadija, Talk given at PENTAQUARK04; http://www.renp. osaka-u.ac.jp/ppenta04/talk/22/Kadija.ppt.

[11] D. Diakonov and V. Petrov, Phys. Rev. D 69, 094011 (2004).

[12] C. E. Carlson, C. D. Carone, H. J. Kwee, and V. Nazaryan, Phys. Lett. B573, 101 (2003).

[13] C. E. Carlson, C. D. Carone, H. J. Kwee, and V. Nazaryan, Phys. Lett. B579, 52 (2004).

[14] R. L. Jaffe and F. Wilczek, Phys. Rev. Lett. 91, 232003 (2003).

[15] Y.-s. Oh, H.-c. Kim, and S. H. Lee, Phys. Rev. D 69, 094009 (2004).

[16] T. D. Cohen, Phys. Rev. D 70, 074023 (2004).

[17] S. Pakvasa and M. Suzuki, Phys. Rev. D 70, 036002 (2004).

[18] P. Bicudo and G. M. Marques, Phys. Rev. D 69, 011503 (2004).

[19] T. Kishimoto and T. Sato, hep-ex/0312003.

[20] P. Bicudo, Phys. Rev. D 70, 111504 (2004).

[21] F. Huang, Z. Y. Zhang, and Y. W. Yu, hep-ph/0411222.

[22] F. J. Llanes-Estrada, E. Oset, and V. Mateu, Phys. Rev. C 69, 055203 (2004).

[23] N. I. Kochelev, H. J. Lee, and V. Vento, Phys. Lett. B594, 87 (2004).
[24] H.-C. Kim, C.-H. Lee, and H.-J. Lee, hep-ph/0402141.

[25] V. Mohta, Phys. Rev. D 70, 114022 (2004).

[26] D. Cabrera, Q. B. Li, V. K. Magas, E. Oset, and M. J. V. Vacas, Phys. Lett. B608, 231 (2005).

[27] H. Nagahiro, S. Hirenzaki, E. Oset, and M. J. V. Vacas, nuclth/0408002.

[28] Particle Data Group, S. Eidelman et al., Phys. Lett. B592, 1 (2004).

[29] T. Hyodo, A. Hosaka, E. Oset, A. Ramos, and M. J. Vicente Vacas, Phys. Rev. C 68, 065203 (2003).

[30] F. E. Close and J. J. Dudek, Phys. Lett. B586, 75 (2004).

[31] T. Hyodo, A. Hosaka, and E. Oset, Phys. Lett. B579, 290 (2004).

[32] W. Liu and C. M. Ko, Phys. Rev. C 68, 045203 (2003).

[33] Y. Oh, H. Kim, and S. H. Lee, Phys. Rev. D 69, 074016 (2004).

[34] Y.-s. Oh, H.-c. Kim, and S. H. Lee, Phys. Rev. D 69, 014009 (2004).

[35] K. Imai, Talk given at YITP Workshop on "Multi-quark Hadrons; Four, Five and More?" Feb. 17-19, 2004; http://www2.yukawa. kyoto-u.ac.jp/rmquark04/files/18/mq04_Imai.ppt.

[36] K. Miwa, Talk given at PENTAQUARK04; http://www.renp. osaka-u.ac.jp/ppenta04/talk/21A/Miwa.ppt.

[37] G. Ecker, Prog. Part. Nucl. Phys. 35, 1 (1995).

[38] A. Pich, Rep. Prog. Phys. 58, 563 (1995).

[39] V. Bernard, N. Kaiser, and U.-G. Meissner, Int. J. Mod. Phys. E4, 193 (1995).

[40] Y. Oh and H. Kim, Phys. Rev. D 70, 094022 (2004).

[41] P. Ko, J. Lee, T. Lee, and J.-h. Park, Phys. Lett. B611, 87 (2005).

[42] Y. R. Liu, A. Zhang, P. Z. Huang, W. Z. Deng, X. L. Chen, and S. L. Zhu, Phys. Rev. D 70, 094045 (2004).

[43] E. Oset and A. Ramos, Nucl. Phys. A635, 99 (1998).

[44] Ya. B. Zeldovich and A. D. Sakharov, Yad. Fiz. 4, 395 (1966); Sov. J. Nucl. Phys. 4, 283 (1967); H. Lipkin, hep-ph/0409349, and references therein.

[45] R. A. Arndt, Y. I. Azimov, M. V. Polyakov, I. I. Strakovsky, and R. L. Workman, Phys. Rev. C 69, 035208 (2004). 